Meike OHLENDORF, Braunschweig

\title{
Zur Phase Rückschau im Problemlöseunterricht
}

In Bezug auf die Förderung der mathematischen Problemlösekompetenz werden in der wissenschaftlichen Literatur bestimmte Aspekte, Sachverhalte oder Vorgänge beim Lösen mathematischer Probleme herausgestellt.

Ein Aspekt, der meist nur in theoretischen Abhandlungen thematisiert wird, ist in diesem Zusammenhang die Phase Rückschau im Pólyaschen Sinne.

\section{Theoretische Grundlagen}

In klassischen Verlaufsmodellen wie z.B. bei Pólya (1945), bei Mason et al. (1982) oder bei Schoenfeld (1985) schließt sich die Phase Rückschau als Abschluss der Problemlösetätigkeiten an das Ausführen eines Planes an.

Die Phase Rückschau wird von Pólya durch den Fragenkatalog in Abbildung 1 charakterisiert. Mason et al. unterteilen die Phase Rückschau in die drei Bereiche Test, Nachdenken und Verallgemeinern.

Polya (1945)
Kannst du das Resultat kontrollieren?
Kannst du den Beweis kontrollieren?
Kannst du das Resultat auf
verschiedene Weise ableiten? Kannst
du es auf den ersten Blick sehen?
Kannst du das Resultat oder die
Methode für irgendeine andere
Aufgabe verwenden?

Mason et al. (1982)

Test:

- Berechnungen

- Überprüfen der Logik

- Plausibiliätskontrollen

- Ist die Aufgabe gelöst?

Nachdenken:

- über die entscheidenden Ideen

- über die Konsequenzen der Lösung

Verallgemeinern:

- Stellen Sie die Lösung in einen größeren Zusammenhang!

- Suchen Sie neue Lösungswege!

- Ändern Sie die Voraussetzungen ab!

\section{Abbildung 1}

Das nichtlineare Modell von Fernandez, Hadaway und Wilson (1994) betont die zyklische Struktur von Problemlöseprozessen und nimmt Managementprozesse wie Selbstkontrolle und Selbststeuerung mit auf. Anders als bei den (in Bezug auf die Phase Rückschau) eher linear angelegten Modellen wie z.B. von Pólya oder Mason et al. wird hier deutlich, dass die Phase Rückschau auch in der Verstehens-, der Planungs- oder der Durchführungsphase auftreten kann. Diese Bedeutungsweiterung der Pólyaschen Rückschau kann außerdem bedeuten, dass sich die Phase Rückschau auch auf falsche oder unvollständige Lösungsversuche beziehen kann.

In Institut für Mathematik und Informatik Heidelberg (Hrsg.), Beiträge zum Mathematikunterricht 2016 (S. x-y). Münster: WTM-Verlag 


\section{Argumente zur Untersuchung der Phase Rückschau im Unterricht}

Mason et al. (1982) bezeichnen die Phase Rückschau als die am meisten vernachlässigte und doch lehrreichste Phase. Kluwe (1981) und Dörner (1982) stellen heraus, dass es beim Problemlösen vor allem auf die dauernde Reflexion des eigenen Problemlöseprozesses ankommt.

Leong et al. (2011) betonen, dass die Phase Rückschau ein Bindeglied zwischen dem Problemlösen und dem (im Vergleich zum Problemlösen) relativ neuen Bereich des Problemstellens darstellt. Dieser Zusammenhang wird insbesondere im zyklischen Verlaufsmodell nach Fernandez/Hadaway/Wilson (1994) deutlich.

In Bezug auf das Lernen mathematischen Problemlösens beklagt Schoenfeld (1985), dass die Rückschau von Schülern zu ihrem Nachteil viel zu selten durchgeführt wird. Er hat außerdem den Eindruck, dass die meisten Lehrer unter der Phase Rückschau nur die Kontrolle der Lösungen verstehen und keine weitergehenden Überlegungen anstellen (Mail an Meike Ohlendorf, 2015).

In einer empirischen Erkundungsstudie zur Gestaltung des Mathematikunterrichts im Grundschulbereich von Heinrich/Pawlitzki/Schuck (2013) wird die Notwendigkeit einer weiteren Befassung mit der Umsetzung der Phase Rückschau im unterrichtlichen Problemlösen unterstrichen. Heinrich/Pawlitzki/Schuck teilen die beobachteten Unterrichtsstunden in drei Problemlösephasen ein, welche sie (I) Präsentation und Verstehen des Problems, (II) Suchen und Finden der Lösungen und (III) Präsentation und Auseinandersetzung mit dem Lösungsgeschehen nennen. Die Befunde zur Phase (III), welche auf der Analyse 16 beobachteter Unterrichtsstunden in Klasse 4 basieren, zeigen, dass in Phase (III) jeweils in Durchschnitt bei einem Viertel bis einem Drittel der Lehrpersonen folgende Maßnahmen vorkamen:

- Nachbereiten, Bewusstmachen von verwendeten Lösungsansätzen

- Auseinandersetzen mit Schwierigkeiten, Fehlern, ungeeigneten Lösungsansätzen

- Eingehen auf alternative Vorgehensweisen, Lösungswege

- Fortführung der Arbeit, weiterführende Probleme

Insgesamt fiel der Anteil der Maßnahme „Fehler“ dabei noch etwas höher aus. Heinrich/Pawlitzki/Schuck betonen, dass eine Bestätigung der Befunde in größerem Ausmaß noch aussteht. 


\section{Konzipierung, Durchführung und erste Befunde der Vorstudie}

In diesem Zusammenhang haben wir im Frühjahr 2015 im Raum Braunschweig eine Erkundungsstudie im Sekundar-I-Bereich an Realschulen und Integrierten Gesamtschulen in den Klassenstufen 9/10 durchgeführt. Die teilnehmenden 6 Lehrer erhielten in dieser Vorstudie zwei Wochen vor Unterrichtsdurchführung ein geometrisches Problem inkl. möglicher Lösungswege. In der didaktischen und methodischen Umsetzung des Problems waren sie frei. Vor der 45-minütigen Unterrichtsstunde wurden die Lehrenden zu Zielen und Ablauf der Stunde befragt, nach der Stunde hatten sie Gelegenheit über den Unterrichtsverlauf zu reflektieren. Das Unterrichtsgeschehen und die Interviews wurden gefilmt, protokolliert und transkribiert. Die Auswertung der Daten erfolgte mithilfe der Methode der konsensuellen Validierung nach Maier (1991). Im Wechselspiel zwischen kategoriengeleitetem und kategorienentwickelndem Vorgehen soll dabei ein System entwickelt werden, mithilfe dessen man Verläufe und wichtige Komponenten unterrichtlichen Problemlösens erfassen kann.

Als vorläufige Befunde der Vorstudie in Bezug auf Phase (III) der beobachteten Unterrichtsstunden lassen sich folgende Erkenntnisse festhalten:

Die meistgewählte Maßnahme in Phase (III) war das Besprechen eines Lösungsweges, die Besprechung erfolgte dabei meist im Unterrichtsgespräch oder durch Schülervorträge. Schwierigkeiten bzw. Fehler wurden dabei kaum, weiterführende Probleme gar nicht thematisiert. Es gab zudem Lehrer, die starke Unsicherheiten zeigten, wenn es darum ging die Richtigkeit von Schülerlösungen einzuschätzen. Lehrer schätzten Schülerlösungen in diesem Zusammenhang falsch ein oder unterschätzten Lösungsansätze von Schülern möglicherweise, weil sie nicht ihrem eigenen favorisierten Lösungsweg entsprachen.

Als vorläufige Schlussfolgerung kann man festhalten, dass es noch Potential gibt, die Vielfalt von Lösungswegen ausgiebiger zu thematisieren. Außerdem unterstreicht die Vorstudie die Bedeutung einer gründlichen fachlichen Vorbereitung für eine erfolgreiche Umsetzung der Phase Rückschau und einen erfolgreichen Problemlöseunterricht.

Die vorläufigen Befunde der Vorstudie unterscheiden sich deutlich von den Ergebnissen der Studie von Heinrich/Pawlitzki/Schuck (2013) im Grundschulbereich.

\section{Ausblick auf die Hauptstudie}

In der Hauptstudie, welche im Frühjahr/Sommer 2016 stattfindet, soll deshalb Mathematikunterricht von 25 Lehrkräften in den Klassen 9/10 an 
Gymnasien gefilmt werden, die ein vorgegebenes geometrisches Problem unterrichten. Das methodische Vorgehen aus der Vorstudie bleibt bis auf kleinere Änderungen erhalten.

Zusätzlich zu den aus der Vorstudie bekannten technischen Hilfsmitteln wird die Lehrkraft in der Hauptstudie mit einer GoPro-Actionkamera mit Brustgurt ausgestattet um die Lehrer-Schüler-Interaktion besser beobachten zu können. Außerdem sollen die Lehrer vor der Stunde einen tabellarischen Unterrichtsverlaufsplan abgeben um die Planung des Lehrers besser nachvollziehen zu können.

Zudem wurde die Unterrichtszeit der Problemlösestunde von 45 Minuten auf 90 Minuten erhöht, da in der Vorstudie nach 45 Minuten oft der Problemlöseprozess noch nicht abgeschlossen war. Der Zielaspekt des Problemlösens wurde in den Informationsmaterialien für die Lehrer klar ins Zentrum gestellt, um die Problemlösestunden bezüglich dieses Aspekts im Vorhinein zu normieren.

Es wird interessant sein zu sehen, ob sich die Befunde der Vorstudie in größerem Umfang bestätigen.

\section{Literatur}

Dörner, D. (1982). Lernen des Wissens- und Kompetenzerwerbs. In B. Treiber \& F.E. Weinert (Hrsg.), Lehr- und Lernforschung (S. 134-148). München: Urban \& Schwarzenberg.

Fernandez M. L., Hadaway, N. \& Wilson, J. W. (1994). Problem solving: Managing it all. The Mathematics Teacher, 87 (3), 195 - 199.

Heinrich, F., Pawlitzki, A. \& Schuck, L. (2013). Problemlöseunterricht in der Grundschule. In F. Käpnick, G. Greefrrath und M. Stein (Hrsg.), Beiträge zum Mathematikunterricht 2013 (S. 448-451). Münster: WTM.

Kluwe, R.H. (1981). Kontrolle eigenen Denkens und Unterricht. In B. Treiber \& F.E. Weinert (Hrsg.), Lehr- und Lernforschung (S. 113-133). München: Urban \& Schwarzenberg.

Maier, H. (1991). Interpretative Forschung im Bereich der Mathematikdidaktik. In: Beiträge zum Mathematikunterricht 1991 (S. 97 - 107). Bad Salzdetfurth: Franzbecker.

Mason, J., Burton, L. \& Stacey, K. (1982). Thinking mathematically. Bristol: AddisonWesley.

Pólya, G. (1945). How to solve it. Princeton: Princeton University Press.

Schoenfeld, A. (1985). Mathematical problem solving. New York: Academic Press.

Schoenfeld, A. (2015). Re: polyas stage looking back. email: alans@berkeley.edu (1503-27)

Leong, Y. H., Tay, E. G., Toh, T. L., Quek, K.S., \& Dindyal, J. (2011). Reviving Pólya's "Look Back" in a Singapore school. Journal of Mathematical Behavior, 30 (3), 181-193. 